\title{
Throughput-Optimal Link-Layer Design in Power Constrained Hybrid OW/RF Systems
}

\author{
Nestor D. Chatzidiamantis, Member, IEEE, Leonidas Georgiadis, Senior Member, IEEE, \\ Harilaos G. Sandalidis, and George K. Karagiannidis, Fellow, IEEE
}

\begin{abstract}
The aim of this paper is to develop link layer transmission schemes for hybrid optical wireless $(\mathrm{OW}) /$ radio frequency (RF) systems, with constraints on both per-link and total average power consumption at the transmitter. In this context, we adopt a timeslot structure with a queue for storing the data packets for transmission and model the hybrid channel as an erasure channel with parameters varying along the time-slots according to a Markov chain. Then, a stochastic optimization problem is formulated, where intelligent decisions regarding the number of the packets admitted in the queue and the power levels used in every link, are taken by the hybrid transmitter in each slot. The objective of this formulation is to design a control policy that maximizes the transmitter throughput, while satisfying the power constraints as well. A solution is offered by using the Lyapunov optimization framework and an on-line transmission algorithm is developed. The proposed transmission algorithm takes decisions based only on the status of the queue and the statistical parameters of the OW/RF channel in each time-slot, without requiring any knowledge of the underlying Markov chain of the channel process, or the statistics of the packet arrival process. Furthermore, in order to alleviate the requirement for full feedback at the transmitter, i.e., feedback for every successfully received packet, which is critical for the accurate queue update, we extend our analysis and incorporate reduced-feedback coding schemes. The proposed transmission policy in this scenario still meets the throughput objective and satisfies the power consumption constraints, while a tradeoff between transmission delays and feedback requirements is revealed.
\end{abstract}

Index Terms-Hybrid optical wireless/radio frequency systems, link layer design, Lyapunov optimization, power constraints, throughput maximization, reduced feedback coding.

Manuscript received May 26, 2014; revised November 7, 2014; accepted April 24, 2015. Date of publication May 12, 2015; date of current version August 17, 2015. Part of this paper was presented at the IEEE International Conference on Communications, Sydney, Australia, June 2014. This research was supported in part by the European Union (European Social Fund ESF) and Greek National Funds through the Operational Program "Education and Lifelong Learning" of the National Strategic Reference Framework (NSRF)Research Funding Program: Thales. Investing in Knowledge Society Through the European Social Fund.

N. D. Chatzidiamantis and L. Georgiadis are with the Department of Electrical and Computer Engineering, Aristotle University of Thessaloniki, Thessaloniki 54 124, Greece (e-mail: nestoras@auth.gr; leonid@ auth.gr).

H. G. Sandalidis is with the Department of Computer Science and Biomedical Informatics, University of Thessaly, Lamia 35 100, Greece (e-mail: sandalidis@dib.uth.gr).

G. K. Karagiannidis is with the Department of Electrical and Computer Engineering, Aristotle University of Thessaloniki, Thessaloniki 54 124, Greece, and also with the Department of Electrical and Computer Engineering, Khalifa University, Abu Dhabi 127788, UAE (e-mail: geokarag@auth.gr).

Digital Object Identifier 10.1109/JSAC.2015.2432512

\section{INTRODUCTION}

$\mathbf{O}$ PERATING at unlicensed optical frequencies, optical wireless (OW) systems offer the potential of broadband capacity in low cost and over distances of up to a few kilometers [1], [2]. However, despite their significant advantages, OW systems are susceptible to atmospheric effects which limit their performance. A prominent way that has been suggested for combating these effects is to use a license-free radio frequency (RF) channel in conjunction with an OW channel [3]. The incentive for implementing such hybrid OW/RF systems is that the two channels are not affected in the same way by atmospheric effects. Specifically, several studies [3]-[5] have shown that fog is the main degrading factor in OW links, while rain does not significantly affect the performance. On the contrary, RF links are susceptible to heavy rain conditions and oxygen absorption, whereas fog has no particular effect.

\section{A. Related Work-Motivation}

Capitalizing on the complementary behaviour of RF and OW channels, multiple transmission techniques suitable for hybrid OW/RF systems have been reported (see [6] and the references therein). However, only few of them dealt with link layer issues, i.e., how to efficiently transmit data streams, which are divided into packets and protected via a cyclic redundancy check (CRC) code, through the hybrid OW/RF channel. In [3], a commercially available transmission technique was presented where the same packets are transmitted through both links but only the packets that are deemed more reliable are kept at the receiver. Despite its minimal feedback requirements (when the packets are corrupted from both links), this scheme does not fully exploit the hybrid OW/RF channel, since the maximum achieved throughput is limited to the higher throughput between the two links. In order to address this issue, an efficient packet coding scheme based on Raptor codes was implemented in [7]. By continuously sending encoded packets through both OW and RF links, this coding scheme was shown to increase the achievable throughput near the capacity limits of the hybrid OW/RF channel, requiring limited feedback. However, any average transmission power constraints that may exist at the hybrid transmitter are not considered in [7].

The assumption of unlimited power consumption at the transmitter is not always valid. For example, power restriction issues arise when hybrid OW/RF links are used in wireless sensor networks [8], [9], or for the communication between moving entities (such as unmanned aerial vehicles, HAPs or satellites) 


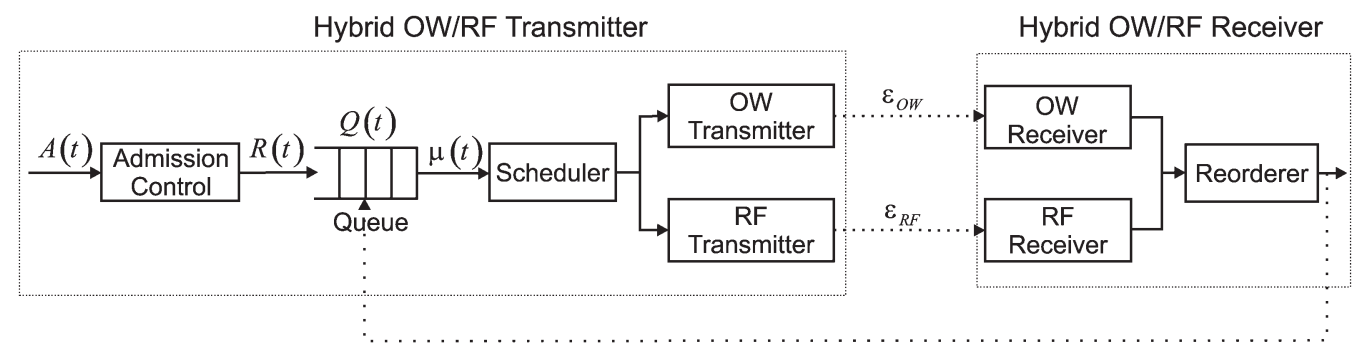

Feedback

Fig. 1. The system model under consideration.

and fixed-point stations [10], [11]. In this case, not only the restrictions on the maximum average transmitted power of each link, imposed from hardware constraints and safety regulations, but also the limitations on the total average power consumption of the transmitter, must be taken into account.

\section{B. Contribution}

In this paper, we propose link-layer transmission policies that can be applied in hybrid OW/RF transmitters with constraints on the average power consumption. To the best of the authors' knowledge, it is the first time that such a design is proposed in hybrid OW/RF systems and can be efficiently applied in the case of power constrained hybrid transmitters. In this context, we assume a timeslot structure together with a queue for storing the data packets for transmission and model the hybrid OW/RF channel as an erasure channel with statistical parameters varying along the time-slots according to a Markov chain. Then, we seek transmission policies that take intelligent per-slot control decisions at the transmitter, regarding the number of the packets admitted in the queue and the power levels used in each link. The objective is to maximize the achievable throughput, while satisfy both the per-link transmission and total power constraints imposed to the hybrid transmitter.

In particular, the contributions of the paper are summarized as follows:

- A solution to the above mentioned stochastic optimization problem is offered using the framework of Lyapunov optimization techniques [12], [13]. An on-line control algorithm is developed that dynamically adapts the control decisions by observing the queue size and the statistical parameters of the OW/RF channel in each time-slot. The main advantage of the developed transmission policy is that it does not require any knowledge of the underlying Markov chain of the channel process, which in practice may be hard or impossible to be obtained. Its performance is investigated in terms of throughput and average queue backlog, and analytical bounds are derived which illustrate its throughput optimality.

- In order to alleviate the requirement of full feedback at the transmitter, i.e., feedback for every successfully received packet, necessary for the accurate update of the queue size, the proposed transmission policy is modified to include the reduced-feedback coding schemes, where linear combinations of the data packets are transmitted instead of the actual data packets [7], [14]-[16]. The difficulties in the design of such transmission algorithms stem from the fact that the queue is not updated in every slot, but only when feedback is received. In addition, the hybrid system model under consideration, which involves the concurrent use of two parallel links with totally different characteristics (data rates and erasure probabilities), introduces extra complexity to this design. The proposed policy addresses such issues and its performance is investigated in terms not only of throughput and average queue backlog, but feedback requirements as well. Analytical bounds for these metrics are derived, which reveal the throughput optimality and the tradeoff between feedback requirements and average queue backlog.

\section{Structure}

The remainder of the paper is organized as follows. In Section II, the system model is introduced with details on the system structure and the channel model. In Section III, the stochastic optimization problem that arises is formulated and the Lyapunov optimization methodology along with the proposed transmission algorithm and its performance bounds, are presented. In Section IV, the modified policy that includes reduced-feedback coding schemes is described and its performance along with certain implementations issues are investigated. Numerical results are illustrated in Section V and, finally, concluding remarks are provided in Section VI.

\section{System And Channel Model}

\section{A. Transceiver Design}

The system model structure is depicted in Fig. 1. In particular, we consider a hybrid OW/RF transmitter, which is composed of an admission control mechanism that determines which packets will be transmitted or discarded, a queue which stores the packets to be transmitted, a scheduler that divides the incoming sequence of packets into two subsequences, according to a predefined pattern depending on the data rates supported by each transmission subsystem, and the OW and RF transmission subsystems used for the packet transmission. At the receiver end, the packets received correctly by each subsystem, are reordered and the original packet sequence is composed. Since correctly received packets are determined 
through the CRC code applied to each packet, both OW and $\mathrm{RF}$ links can be modeled as erasure channels with erasure probabilities $\epsilon_{O W}$ and $\epsilon_{R F}$, respectively [7]. Next, we discuss how these probabilities can be calculated by the transmitter, based on some known system and channel parameters.

\section{B. Timeslot Structure}

We assume that the transmitter operates over a slot based structure. Namely, the timeline is divided into successive nonoverlapping slots, where the slot is denoted as $t=0,1,2, \ldots$ and corresponds to the time interval $[t, t+1) ; t$ and $t+1$ are called the "beginning" and the "end" of the slot, respectively. During slot $t$, new packets arrive at the transmitter according to an independent and identically distributed (i.i.d.) arrival process $A(t)$ with mean rate $\lambda$ packets per slot and $A(t) \leq A_{\max }$, where $A_{\max }$ is a finite constant.

The parameters affecting the channel erasure probabilities or equivalently the successful packet transmission, compose the channel state $s$, which is defined in the Section II-D. The channel state process $s(t)$ remains constant within a time slot, and evolves according to a general homogeneous, aperiodic and irreducible discrete time Markov chain with a finite state space $\mathcal{S}$. Hence, the stationary distribution of $s(t)$ is given by

$$
\operatorname{Pr}(s(t)=i)=\pi_{i},
$$

where $\pi_{i}$ represents the steady state probability distribution of the Markov chain over states $i \in \mathcal{S}$.

\section{Power Constraints}

Let $P_{1}(t)$ and $P_{2}(t)$ denote the power levels used for transmission in the OW and RF subsystems in slot $t$. These power allocations are chosen from the sets $\mathcal{P}_{1}$ and $\mathcal{P}_{2}$, satisfying maximum instantaneous power constraints imposed from hardware limitations. Hence,

$$
P_{1}(t) \leq P_{1, \max } \text { and } P_{2}(t) \leq P_{2, \max },
$$

where $P_{1, \max }$ and $P_{2, \max }$ represent the maximum power levels used for transmission in OW and RF subsystems respectively, imposed by safety regulations [17, Sec. 2.1.2]. Furthermore, we seek transmission policies that select appropriate power allocations subject to the limitation on the total average power consumption of the hybrid transmitter. To this end, the following constraint needs to be satisfied

$$
\lim \sup _{t \rightarrow \infty} \frac{1}{t} \sum_{\tau=0}^{t-1} \mathbb{E}\left\{f_{1}\left(P_{1}(\tau)\right)+f_{2}\left(P_{2}(\tau)\right)\right\} \leq \hat{P},
$$

where $\hat{P}$ is the average electrical power consumed by hybrid transmitter and $\mathbb{E}\{\cdot\}$ denotes expectation. In addition, $f_{1}(\cdot)$ and $f_{2}(\cdot)$ are non-decreasing functions with respect to the transmission power levels, that determine the electrical power consumed by the OW and RF subsystems, respectively. In general, the structure of these functions depends on the technology employed by each transmission subsystem. A relevant measurement campaign, regarding the total power consumption of
OW and RF transmission subsystems for specific transmission powers and technologies, has been performed in [8].

\section{Channel Model}

Due to the insertion of the CRC in every packet, the erasure channel model is used for describing both OW and RF channels. By erasure, we define the event that the received power is below a pre-defined threshold which determines whether a transmitted packet is successfully received or not [3]. The probabilities of the erasure events for each link depend on the power level used in each link and the state of the hybrid OW/RF channel which is defined as

$$
s=\left\{L, v, C_{n}^{2}, \alpha_{\text {oxy }}, \alpha_{\text {rain }}, K\right\},
$$

where $L$ is the link distance (in $\mathrm{km}$ ), $v$ and $C_{n}^{2}$ are the weather dependent attenuation coefficient (in $1 / \mathrm{km}$ ) and the refractiveindex structure parameter of the OW link, $\alpha_{\text {oxy }}$ and $\alpha_{\text {rain }}$ are the attenuation coefficients for the RF link caused by oxygen absorption and rain respectively, and $K$ is the fading parameter of the RF link. ${ }^{1}$ Next, we describe the mode of operation of the OW and RF transmission subsystems and derive the erasure probabilities for each link as a function of the channel state and the transmission power levels.

1) Transmission Through the OW Subsystem: We consider an OW subsystem which employs intensity modulation at the transmitter and direct detection at the receiver. Assuming $P_{1}$ as the transmitted optical power level and $s$ as the channel state, the erasure probability of the OW link is given by

$$
\epsilon_{O W}\left(P_{1}, s\right)=\operatorname{Pr}\left\{h_{1} P_{1} \leq P_{\text {sens }}\right\}=\operatorname{Pr}\left\{h_{1} \leq \frac{P_{\text {sens }}}{P_{1}}\right\},
$$

where $h_{1}$ denotes the channel gain of the OW link, while $P_{\text {sens }}$ is the sensitivity of the photodetector which ensures that CRC is successfully decoded.

Due to atmospheric effects, the channel gain of the OW link can be modeled as

$$
h_{1}=\bar{h}_{1} \tilde{h}_{1},
$$

where $\bar{h}_{1}$ accounts for path loss due to weather effects and geometric spread loss, and $\tilde{h}_{1}$ represents irradiance fluctuations caused by atmospheric turbulence. The path loss coefficient can be calculated by combining the Beer Lambert's law [1] with the geometric loss formula [7, Eq. (9)], yielding

$$
\bar{h}_{1}=\left(\operatorname{erf}\left(\frac{\sqrt{\pi} D}{\sqrt{2} \theta_{T} L}\right)\right)^{2} \exp (-v L)
$$

where $\operatorname{erf}(\cdot)$ is the error function, $2 D$ is the aperture diameter and $\theta_{T}$ is the optical beam's divergence angle (in $m \mathrm{rad}$ ). Under a wide range of atmospheric conditions, turbulence induced fading is statistically described by the well known GammaGamma distribution, whose atmospheric parameters $\alpha$ and $\beta$

\footnotetext{
${ }^{1}$ To facilitate the analysis, we assume that $s$ is limited to a finite set of practical values, i.e., $\mathcal{S}$ is assumed to be a finite state space.
} 
are calculated based on $L, C_{n}^{2}$ and the wavelength of the optical carrier $\lambda_{1}[1]$ according to

$$
\alpha=\left(\exp \left(\frac{0.49 \sigma_{R}^{2}}{\left(1+1.11 \sigma_{R}^{\frac{12}{5}}\right)^{\frac{7}{6}}}\right)-1\right)^{-1}
$$

and

$$
\beta=\left(\exp \left(\frac{0.51 \sigma_{R}^{2}}{\left(1+0.69 \sigma_{R}^{\frac{12}{5}}\right)^{\frac{7}{6}}}\right)-1\right)^{-1},
$$

where $\sigma_{R}^{2}$ is the Rytov variance given by

$$
\sigma_{R}^{2}=1.23 C_{n}^{2}\left(\frac{2 \pi}{\lambda_{1}}\right)^{\frac{7}{6}} L^{\frac{11}{6}}
$$

Based on the above and using [18, Eq. (7)], the erasure probability of the OW link can be analytically evaluated by

$$
\epsilon_{O W}\left(P_{1}, s\right)=\frac{1}{\Gamma(\alpha) \Gamma(\beta)} G_{1,3}^{2,1}\left[\frac{\alpha \beta P_{\text {sens }}}{\bar{h}_{1} P_{1}} \mid \begin{array}{c}
1 \\
\alpha, \beta, 0
\end{array}\right],
$$

where $\Gamma(\cdot)$ is the Gamma function [19, Eq. (8.310)] and $G_{p, q}^{m, n}[\cdot]$ is the Meijer's $G$-function [19, Eq. (9.301)].

2) Transmission Through the RF Subsystem: We will assume that the RF transmission subsystem uses a line-of-sight (LOS) link in the millimeter wave band. If $P_{2}$ is the transmitted $\mathrm{RF}$ power, the erasure probability of the RF link for a given channel state $s$, is defined as

$$
\epsilon_{R F}\left(P_{2}, s\right)=\operatorname{Pr}\left\{\gamma_{R F} \leq \gamma_{t h}\right\},
$$

where $\gamma_{R F}$ is the instantaneous signal-to-noise ratio (SNR) at the $\mathrm{RF}$ receiver and $\gamma_{t h}$ is a threshold that ensures no errors are detected by the CRC. The instantaneous SNR of the RF link is defined as

$$
\gamma_{R F}=\frac{P_{2} g E_{s}}{\sigma_{2}^{2}} h_{2}^{2},
$$

where $h_{2}$ is the fading gain of the RF link, $g$ is the average path loss, $P_{2}$ is the transmitted RF power, $E_{s}$ is the average energy of the modulation alphabet, and finally $\sigma_{2}^{2}$ is the variance of the zero mean circularly symmetric complex Gaussian noise.

Assuming a carrier frequency of $60 \mathrm{GHz}$, the average path loss attenuation of the RF link is given by [20, Eq. (7)]

$$
g[d B]=G_{t}+G_{R}-20 \log _{10}\left(\frac{4 \pi L}{\lambda_{2}}\right)-\alpha_{o x y} L-\alpha_{\text {rain }} L,
$$

where $G_{t}$ and $G_{i}$ denote the transmit and receive antenna gains, respectively and $\lambda_{2}$ is the wavelength of the RF system. Furthermore, due to the LOS characteristic, the fading gain, $h_{2}$, is Ricean distributed [20], with the fading parameter, $K$, depending on various link parameters, such as link distance and antenna height.

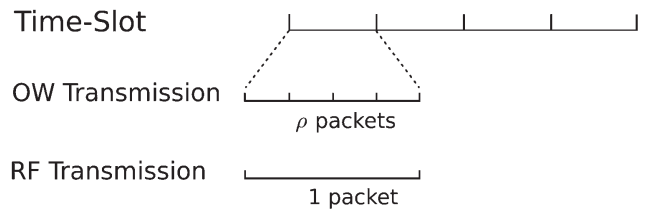

Fig. 2. The number of packets transmitted within a time slot.

Based on the above and using [21, pp. 420], the erasure probability of the RF link can be analytically evaluated by

$$
\epsilon_{R F}\left(P_{2}, s\right)=1-\mathbb{Q}_{1}\left(\sqrt{2 K}, \sqrt{\frac{2(1+K) \gamma_{t h}}{\frac{P_{2} g E_{s}}{\sigma_{2}^{2}}}}\right),
$$

where $\mathbb{Q}_{1}(\cdot, \cdot)$ is the first-order Marcum function [21, Eq. (4.34)].

\section{E. Available Controls and Queueing Dynamics}

Let $Q(t) \in\{1,2,3, \ldots\}$ represent the state of the queue of the hybrid transmitter at slot $t$.

At the beginning of each slot, resource allocation decisions are taken, i.e., $\left(P_{1}(t), P_{2}(t)\right)$ are selected from the sets $\mathcal{P}_{1}$ and $\mathcal{P}_{2}$ respectively. Based on these resource allocation decisions, the number of the packets that depart from the queue of the hybrid transmitter is determined. Assuming full feedback at the transmitter, i.e., feedback for every successfully received packet, a packet is removed from the queue of the hybrid transmitter only when correctly received. Thus, the departures from the queue in each time-slot can be modeled as a random process $\mu(t)$, depending on the channel state and the power levels $P_{1}(t)$ and $P_{2}(t)$ used for transmission by each subsystem. This process can be written as

$$
\mu(t)=\mu_{1}(t)+\mu_{2}(t),
$$

where $\mu_{1}(t)$ and $\mu_{2}(t)$ are the stochastic processes that represent the numbers of packets successfully transmitted from the OW and RF transmission subsystems, respectively, and removed from the queue. These processes can be expressed as $\mu_{1}(t)=\rho \mathbb{1}_{1}\left(P_{1}(t), s(t)\right)$ and $\mu_{2}(t)=\mathbb{1}_{2}\left(P_{2}(t), s(t)\right)$, where $\mathbb{1}_{1}\left(P_{1}(t), s(t)\right)$ and $\mathbb{1}_{2}\left(P_{2}(t), s(t)\right)$ are indicator functions that represent the random success/failure transmission outcomes of each transmission subsystem, when the channel state is $s(t)$ and the optical and RF transmission power levels are $P_{1}(t)$ and $P_{2}(t)$, respectively. Moreover, $\rho$ is a scaling parameter that accounts for the different transmission rates supported by each subsystem, i.e., $\rho=\frac{R_{O W}}{R_{R F}}$, where $R_{O W}$ and $R_{R F}$ are the transmission rates supported by the OW and RF subsystems, respectively. Since in most cases, OW systems can support higher data rates than their RF counterparts, we can assume that $\rho \geq 1$. Moreover to simplify the discussion, we assume that $\rho$ is integer. ${ }^{2}$ A pictorial representation of the difference in data rates between the transmission subsystems is depicted in Fig. 2, where the slot duration is selected so that $R_{R F}=1$ packet/slot.

\footnotetext{
${ }^{2}$ In case that fewer than $\rho+1$ packets exist in the queue at a given time-slot, "dummy" packets are inserted and transmitted.
} 
The probabilities of successful transmission for each subsystem, denoted as $\Phi_{1}\left(P_{1}(t), s(t)\right)$ and $\Phi_{2}\left(P_{2}(t), s(t)\right)$, are assumed to be non-decreasing functions with respect to the transmission powers and are determined through the erasure probabilities of each link, according to

$$
\begin{aligned}
\Phi_{1}\left(P_{1}(t), s(t)\right) & =\mathbb{E}\left\{\mathbb{1}_{1}\left(P_{1}(t), s(t)\right)\right\} \\
& =1-\epsilon_{O W}\left(P_{1}(t), s(t)\right)
\end{aligned}
$$

and

$$
\begin{aligned}
\Phi_{2}\left(P_{2}(t), s(t)\right) & =\mathbb{E}\left\{\mathbb{1}_{2}\left(P_{2}(t), s(t)\right)\right\} \\
& =1-\epsilon_{R F}\left(P_{2}(t), s(t)\right) .
\end{aligned}
$$

At the end of each time-slot, the admission control decision is taken and $R(t)$, i.e., the number of the data packets arrived in the slot and admitted into the queue, is determined, where

$$
0 \leq R(t) \leq A(t)
$$

In view of the above, the queue evolves as [12]

$$
Q(t+1)=[Q(t)-\mu(t)]^{+}+R(t),
$$

where $[x]^{+}=\max [x, 0]$.

\section{Design Objective And Transmission Algorithm}

The major challenge in the design of the hybrid transmitter is to maximize its throughput by taking the appropriate control decisions in each time-slot and ensuring that the average power constraints are satisfied. In what follows, we describe the stochastic optimization problem that arises for the system model under consideration and suggest a solution based on the wellknown Lyapunov optimization framework [12].

\section{A. Design Objective}

Consider any policy that makes admission control $R(t)$ and power allocation $\left(P_{1}(t), P_{2}(t)\right)$ decisions. A policy is called admissible if the queue of the hybrid transmitter is meanrate stable, i.e., the output long-term rate of the queue should be equal with the input rate, the constraint of the maximum number of admissible packets given by (19) is satisfied for every time-slot, and the power constraints of (2), (3) are satisfied. Under an admissible policy, the hybrid transmitter obtains a long-term average transmission throughput

$$
\bar{R}=\lim \inf _{t \rightarrow \infty} \frac{1}{t} \sum_{\tau=0}^{t-1} \mathbb{E}\{R(\tau)\} .
$$

Our goal is to select an admissible policy that maximizes the throughput. By denoting as $\gamma$ the joint admission control and power allocation decision and $\Gamma$ the set that contains every possible possible, this can be stated by the following stochastic optimization problem, named OPT, as

$$
\begin{aligned}
\max \cdot \gamma \in \Gamma & \bar{R} \\
\text { s.t. } & \bar{R} \leq \bar{\mu} \\
& P_{1}(t) \leq P_{1, \max } \text { and } P_{2}(t) \leq P_{2, \max }, \\
& \bar{P}_{\text {tot }} \leq \hat{P}, \\
& 0 \leq R(t) \leq A(t) \quad \forall t \\
& P_{1}(t) \in \mathcal{P}_{1} \text { and } P_{2}(t) \in \mathcal{P}_{2} \quad \forall t,
\end{aligned}
$$

where

$$
\begin{aligned}
\bar{\mu} & =\lim \sup _{t \rightarrow \infty} \frac{1}{t} \sum_{\tau=0}^{t-1} \mathbb{E}\{\mu(t)\}, \\
\bar{P}_{t o t} & =\lim \sup _{t \rightarrow \infty} \frac{1}{t} \sum_{\tau=0}^{t-1} \mathbb{E}\left\{f_{1}\left(P_{1}(\tau)\right)+f_{2}\left(P_{2}(\tau)\right)\right\}
\end{aligned}
$$

and $f_{1}(\cdot)$ and $f_{2}(\cdot)$ are the functions, defined in (3), that relate the electrical power consumption of OW and RF transmission subsystems with the respective transmission powers.

Based on well known results on stochastic network optimization [12], [13], in order to obtain an optimal control policy for OPT, it is sufficient to consider only the class of stationary, randomized policies that take control actions based on a stationary probability distribution over the control action set, ignoring past history. Let $v^{*}$ denote the optimal value of the objective in OPT. Then, we have the following lemma.

Lemma 1: If the set of admissible policies is non-empty, there exists a stationary, randomized policy STAT that takes control decisions in every time slot purely as a (possibly randomized) function of the current channel state $s(t)$, while satisfying the constraints $R^{\text {stat }}(t) \leq A(t), P_{1}^{\text {stat }}(t) \in \mathcal{P}_{1}$ and $P_{2}^{\text {stat }}(t) \in \mathcal{P}_{2}$ for all $t$, and providing the following guarantees:

$$
\begin{aligned}
\bar{R}^{\text {stat }} & =v^{*} \\
\bar{R}^{\text {stat }} & \leq \bar{\mu}^{\text {stat }} \\
\bar{f}_{1}\left(P_{1}^{\text {stat }}\right)+\bar{f}_{2}\left(P_{2}^{\text {stat }}\right) & \leq \hat{P},
\end{aligned}
$$

where $\bar{R}^{\text {stat }}, \bar{\mu}^{\text {stat }}, \bar{f}_{1}\left(P_{1}^{\text {stat }}\right)$ and $\bar{f}_{2}\left(P_{2}^{\text {stat }}\right)$ denote the long-term time averages of the corresponding parameters under this policy.

The above Lemma can be clarified using the following case study.

Example 2: We consider a hybrid OW/RF system with $\mathcal{P}_{1}=$ $\left\{0, P_{1, \max }\right\}, \mathcal{P}_{2}=\left\{0, P_{2, \max }\right\}$ and $\mathcal{S}=\left\{i_{0}\right\}$. A stationary policy takes randomized decisions based on the following probabilities

$$
\begin{aligned}
& x=\operatorname{Pr}\left\{P_{1}(t)=P_{1, \text { max }}, P_{2}(t)=0\right\}, \\
& y=\operatorname{Pr}\left\{P_{1}(t)=0, P_{2}(t)=P_{2, \text { max }}\right\}, \\
& z=\operatorname{Pr}\left\{P_{1}(t)=P_{1, \text { max }}, P_{2}(t)=P_{2, \text { max }}\right\}
\end{aligned}
$$

and

$$
w=\operatorname{Pr}\left\{P_{1}(t)=0, P_{2}(t)=0\right\} .
$$


TABLE I

HYBRID SYSTEM PARAMETERS [3], [20], [26]

\begin{tabular}{|c|c|c|}
\hline \multicolumn{3}{|c|}{ OW sub-system } \\
\hline Parameter & Symbol & Value \\
\hline Data rate & $R_{O W}$ & 4 packets/slot \\
\hline Wavelength & $\lambda_{1}$ & $780 \mathrm{~nm}$ \\
\hline Max optical power & $P_{1, m a x}$ & $40 \mathrm{~mW}$ \\
\hline Aperture diameter & $2 D$ & $10 \mathrm{~cm}$ \\
\hline Divergence angle & $\theta$ & $5 \mathrm{mrad}$ \\
\hline Receiver's Sensitivity & $P_{\text {sens }}$ & $1 \mu \mathrm{W}$ \\
\hline \multicolumn{3}{|c|}{ RF sub-system } \\
\hline Parameter & Symbol & Value \\
\hline Data rate & $R_{R F}$ & 1 packet/slot \\
\hline Carrier frequency & $f_{c}$ & $60 \mathrm{GHz}$ \\
\hline Max transmit power & $P_{2, m a x}$ & $10 \mathrm{~mW}$ \\
\hline Average symbol's energy & $E_{s}$ & $1 \mathrm{~J}$ \\
\hline Transmit/Receive Antenna Gain & $G_{i}, G_{r}$ & $44 \mathrm{dBi}$ \\
\hline Attenuation (oxygen) & $\alpha_{o x y}$ & $15.1 \mathrm{~dB} / \mathrm{km}$ \\
\hline Noise variance & $\sigma_{2}^{2}$ & $-85 \mathrm{dBm}$ \\
\hline Threshold SNR & $\gamma_{t h}$ & $24 \mathrm{~dB}$ \\
\hline Ricean fading parameter & $K$ & $6 \mathrm{~dB}$ \\
\hline
\end{tabular}

Furthermore, the average number of the packets that depart from the queue when this policy is applied, is given by

$$
\begin{aligned}
\bar{\mu}=x \rho \Phi_{1}( & \left.P_{1, \max }, i_{0}\right)+y \Phi_{2}\left(P_{2, \max }, i_{0}\right) \\
& +z\left(\rho \Phi_{1}\left(P_{1, \max }, i_{0}\right)+\Phi_{2}\left(P_{2, \max }, i_{0}\right)\right) .
\end{aligned}
$$

Based on Lemma 1, the optimal stationary policy, i.e., STAT policy, will maximize the average number of both admitted in the queue and transmitted packets, leading to

$$
R^{s t a t}=\mu^{\text {stat }}=v^{*} .
$$

Thus, the admission control decision under this policy is determined as

$$
R^{s t a t}(t)= \begin{cases}A(t) & \text { w.p. } \frac{v^{*}}{\lambda} \\ 0 & \text { else }\end{cases}
$$

resulting in

$$
\bar{R}^{\text {stat }}=\mathbb{E}\{A(t)\} \frac{v^{*}}{\lambda}=v^{*}
$$

Moreover, the control probabilities used by the STAT policy, and $v^{*}$ are calculated according to

$$
\begin{array}{cc}
\max . & x \rho \Phi_{1}\left(P_{1, \max }, i_{0}\right)+y \Phi_{2}\left(P_{2, \max }, i_{0}\right) \\
& +z\left(\rho \Phi_{1}\left(P_{1, \max }, i_{0}\right)+\Phi_{2}\left(P_{2, \max }, i_{0}\right)\right) \\
\text { s.t. } & (x+z) f_{1}\left(P_{1, \max }\right)+(y+z) f_{2}\left(P_{2, \max }\right) \leq \hat{P} \\
x+y+z \leq 1
\end{array}
$$

where $v^{*}$ is the optimum value of the objective function. The above optimization problem is a linear-programming problem that can be efficiently solved through standard algorithms [22]. For the system model parameters provided by Table I and $\hat{P}=90 \mathrm{~mW}$, the values of $x=0, y=0.3$ and $z=0.7$ were obtained from the solution of (30), irrespective of the channel state conditions provided by Table II.
TABLE II

Channel State Parameters [20]

\begin{tabular}{|c|c|c|c|c|c|}
\hline state & $\begin{array}{c}\text { Weather } \\
\text { Conditions }\end{array}$ & $\begin{array}{c}L \\
(\mathrm{~m})\end{array}$ & $\begin{array}{c}v \\
(\mathrm{~dB} / \mathrm{km})\end{array}$ & $\begin{array}{c}\alpha_{\text {rain }} \\
(\mathrm{dB} / \mathrm{km})\end{array}$ & $\begin{array}{c}C_{n}^{2} \\
\left(\mathrm{~m}^{-2 / 3}\right)\end{array}$ \\
\hline \hline$s_{1}$ & Clear air & 1400 & 0.43 & 0 & $5 \cdot 10^{-14}$ \\
\hline$s_{2}$ & Haze & 1400 & 4.2 & 0 & $1.7 \cdot 10^{-14}$ \\
\hline$s_{3}$ & Rain $(12.5 \mathrm{~mm} / \mathrm{h})$ & 1400 & 5.8 & 5.6 & $5 \cdot 10^{-15}$ \\
\hline
\end{tabular}

It should be noted that only a single state in $\mathcal{S}$ is assumed in the above example. In the more general case where there are multiple channel states, the conventional techniques to solve OPT require extensive knowledge of the channel state statistics which may be difficult or impossible to be obtained. In order to avoid such a requirement, we apply the Lyapunov optimization framework [12] that is based only on the observations of the channel state and the queue size in each time slot.

\section{B. Minimizing the Variable-Slot "Drift Plus Penalty"}

In the general Lyapunov optimization framework, a "Drift Plus Penalty" expression is derived which is minimized for every time slot [12], [13]. In our case, since the channel state is modulated by a discrete time Markov-chain, indicating that it is non i.i.d., over the time-slots, we employ a slight variation of this method where a variable-slot "Drift Plus Penalty" expression is minimized. For the optimization problem OPT, this expression is derived in what follows.

Initially, we define the virtual queue, $H(t)$, which evolve as

$$
H(t+1)=[H(t)-\hat{P}]^{+}+f_{1}\left(P_{1}(t)\right)+f_{2}\left(P_{2}(t)\right) .
$$

If the designed control policy makes virtual queue mean rate stable, the long-term average power constraint of (3) is satisfied.

Let $\Theta(t)=\{Q(t), H(t)\}$ represent the system queue backlog at slot $t$ and $L(\Theta(t))$ denote the quadratic Lyapunov function which is defined as

$$
L(\Theta(t))=\frac{1}{2} Q^{2}(t)+\frac{1}{2} H^{2}(t) .
$$

Furthermore, we designate the state $i_{0} \in \mathcal{S}$ as the renewal state of $\mathcal{S}$. Hence, the sequence $\left\{T_{r}\right\}_{r=0}^{\infty}$, that represents the recurrences times to state $i_{0}$, is i.i.d., with $\mathbb{E}\left\{T_{r}\right\}$ and $\mathbb{E}\left\{T_{r}^{2}\right\}$ being finite constants given by [23]

$$
\mathbb{E}\left\{T_{r}\right\}=\mathbb{E}\{T\}=\frac{1}{\pi_{i_{0}}}
$$

and $\mathbb{E}\left\{T_{r}^{2}\right\}=\mathbb{E}\left\{T^{2}\right\}$ for all $r$, where $T$ is a random variable that follows the stationary distribution of $T_{r}$.

Denoting by $t_{r}$ the time of the $r$ th revisitation to state $i_{0}$, where $t_{r}=\sum_{j=0}^{r-1} T_{j}$, we define the variable-slot Lyapunov drift as the expected change in the Lyapunov function from renewal time $t_{r}$ to renewal time $t_{r+1}$ conditioned on $\Theta\left(t_{r}\right)$, i.e.,

$$
\Delta\left(\Theta\left(t_{r}\right)\right)=\mathbb{E}\left\{L\left(\Theta\left(t_{r+1}\right)\right)-L\left(\Theta\left(t_{r}\right)\right) \mid \Theta\left(t_{r}\right)\right\} .
$$

According to the T-slot Drift lemma presented in [12, pp. 73] and using (20) and (31), the variable-slot Lyapunov drift can be 
upper bounded by (35),

$$
\begin{aligned}
\Delta\left(\Theta\left(t_{r}\right)\right) \leq & B \mathbb{E}\left\{T_{r}^{2} \mid \Theta\left(t_{r}\right)\right\} \\
& +Q\left(t_{r}\right) \mathbb{E}\left\{\sum_{\tau=t_{r}}^{t_{r}+T_{r}-1}(R(\tau)-\mu(\tau)) \mid \Theta\left(t_{r}\right)\right\} \\
& +H\left(t_{r}\right) \mathbb{E}\left\{\sum_{\tau=t_{r}}^{t_{r}+T_{r}-1}\left(f_{1}\left(P_{1}(\tau)\right)+f_{2}\left(P_{2}(\tau)\right)-\hat{P}\right) \mid \Theta\left(t_{r}\right)\right\}
\end{aligned}
$$

where $B$ is a finite constant that satisfies for all $t$

$B \geq \frac{1}{2}\left(R^{2}(t)+\mu^{2}(t)\right)+\frac{1}{2}\left(\hat{P}^{2}+\left(f_{1}\left(P_{1}(t)\right)+f_{2}\left(P_{2}(t)\right)\right)^{2}\right)$.

Taking (2) into consideration, the fact that the functions $f_{1}(\cdot)$ and $f_{2}(\cdot)$ are non-decreasing, $R(t) \leq A_{\max }$ and $\mu(t) \leq \rho+1$, it follows that the above equation can be satisfied by choosing

$B=\frac{1}{2}\left(A_{\text {max }}^{2}+(\rho+1)^{2}+\hat{P}^{2}+\left(f_{1}\left(P_{1, \text { max }}\right)+f_{2}\left(P_{2, \text { max }}\right)\right)^{2}\right)$.

By adding the penalty term $-V \mathbb{E}\left\{\sum_{\tau=t_{r}}^{t_{r}+T_{r}-1} R(\tau) \mid \Theta\left(t_{r}\right)\right\}$ to both sides of (35), where $V$ is a utility-delay trade-off parameter, and after some basic algebraic manipulations, the variableslot "Drift Plus Penalty" expression is derived as (38), shown at the bottom of the page.

According to the Markov modulated processes theorem presented in [12, pp. 77], the right-term of Eq. (38) can be minimized by observing $\Theta(t)$ and $s(t)$ in every time slot and opportunistically minimizing the expectations. This is achieved by the Hybrid Transmitter Control (HTC) algorithm, described below.

\section{HTC Algorithm}

In each time-slot $t \in\{1,2,3, \ldots\}$, after observing $\Theta(t)$ and $s(t)$ do the following:

1) Admission Control: Choose the number of admitted packets according to

$$
R(t)= \begin{cases}A(t) & \text { if } Q(t) \leq V \\ 0 & \text { else }\end{cases}
$$

2) Resource Allocation: Choose the power allocation controls $\left(P_{1}(t), P_{2}(t)\right)$ that solve the following optimization problems:

$$
\begin{array}{rr}
\max . & \rho Q(t) \Phi_{1}\left(P_{1}(t)\right)-H(t) f_{1}\left(P_{1}(t)\right) \\
\text { s.t. } & P_{1}(t) \in \mathcal{P}_{1}
\end{array}
$$

and

$$
\begin{array}{rr}
\max . & Q(t) \Phi_{2}\left(P_{2}(t)\right)-H(t) f_{2}\left(P_{2}(t)\right) \\
\text { s.t. } & P_{2}(t) \in \mathcal{P}_{2}
\end{array}
$$

3) Quеие Update: After choosing the appropriate controls, update queues according to (20), (31).

The following theorem returns bounds for the performance of the HTC algorithm, in terms of average transmission throughput and maximum queue size.

Theorem 3: Suppose the HTC algorithm is implemented over all time-slots $t \in\{1,2,3, \ldots\}$ with initial conditions $Q(0)=0$, $H_{1}(0)=H_{2}(0)=H_{3}(0)=0$ and with a control parameter $V>0$. Then:

1) The worst case queue backlog is upper bounded by a constant $Q_{\max }$ for all $t$ given by

$$
Q(t) \leq Q_{\max }=A_{\max }+V,
$$

2) The long-term time average throughput utility achieved by the HTC algorithm is within $O\left(\frac{1}{V}\right)$ of the optimal value, i.e.,

$$
\bar{R} \geq v^{*}-\frac{B \mathbb{E}\left\{T^{2}\right\}}{V \mathbb{E}\{T\}},
$$

where $v^{*}$ denotes the optimal value of the objective in OPT.

Proof: For the first part, suppose that $Q(t) \leq Q_{\max }$ at the slot $t$. We will show that the same holds at the frame $t+1$. If $Q(t) \leq Q_{\max }-A_{\max }$, then from (20) we have $Q(t+1) \leq Q_{\max }$, since $R(t) \leq A(t) \leq A_{\text {max }}$. Furthermore, if $Q(t)>Q_{\max }-A_{\max }=V$, then the admission control part of the HTC algorithm chooses $R(t)=0$, resulting in $Q(t+1) \leq Q(t) \leq Q_{\max }$. The second part can be directly derived by using the results of the Markov modulated processes theorem presented in [12, pp. 77].

Theorem 3 shows that the long-term time-average throughput can be pushed to within $O\left(\frac{1}{V}\right)$ of the optimal value with a trade-off in the worst case queue backlog. By Little's Theorem, this leads to an $O\left(\frac{1}{V}, V\right)$ utility-delay tradeoff.

$$
\begin{aligned}
\Delta\left(\Theta\left(t_{r}\right)\right) & -V \mathbb{E}\left\{\sum_{\tau=t_{r}}^{t_{r}+T_{r}-1} R(\tau) \mid \Theta\left(t_{r}\right)\right\} \leq B \mathbb{E}\left\{T_{r}^{2} \mid \Theta\left(t_{r}\right)\right\}+\left(Q\left(t_{r}\right)-V\right) \mathbb{E}\left\{\sum_{\tau=t_{r}}^{t_{r}+T_{r}-1} R(\tau) \mid \Theta(t)\right\} \\
& -\mathbb{E}\left\{\sum_{\tau=t_{r}}^{t_{r}+T_{r}-1} H\left(t_{r}\right) \hat{P} \mid \Theta\left(t_{r}\right)\right\}+\mathbb{E}\left\{\sum_{\tau=t_{r}}^{t_{r}+T_{r}-1}\left(-Q\left(t_{r}\right) \mu_{2}(\tau)+H\left(t_{r}\right) f_{2}\left(P_{2}(\tau)\right)\right) \mid \Theta\left(t_{r}\right)\right\} \\
& +\mathbb{E}\left\{\sum_{\tau=t_{r}}^{t_{r}+T_{r}-1}\left(-Q\left(t_{r}\right) \mu_{1}(\tau)+H\left(t_{r}\right) f_{1}\left(P_{1}(\tau)\right)\right) \mid \Theta\left(t_{r}\right)\right\}
\end{aligned}
$$


Hybrid OW/RF Transmitter

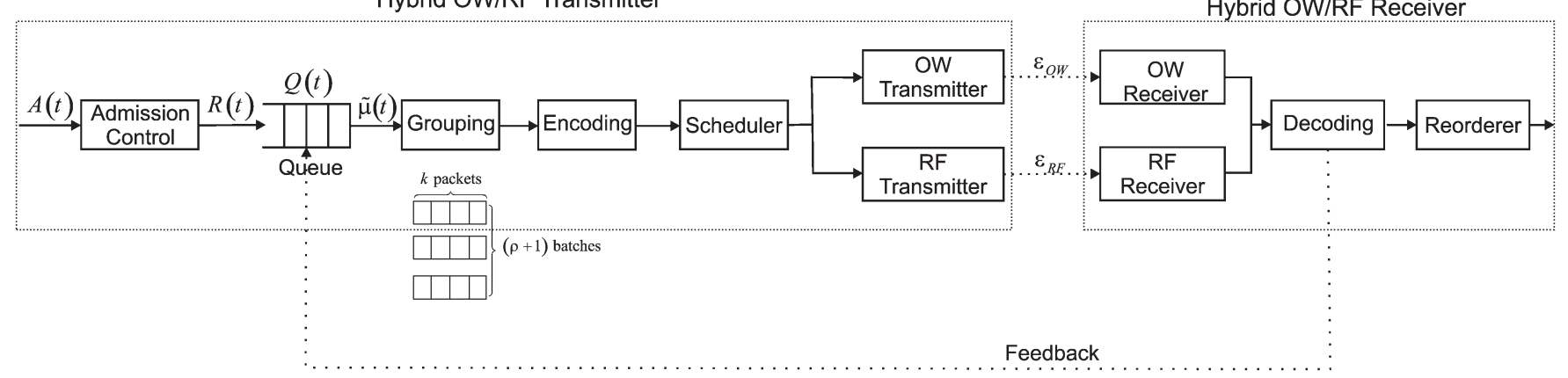

Fig. 3. The modified system model under consideration.

\section{Reduced-FeEdBack Transmission Algorithms}

In the previous sections, we have investigated optimal transmission algorithms for hybrid OW/RF systems when there is full feedback at the transmitter, i.e., the transmitter knows exactly which packets have been received without errors by the receiver. However, this full-feedback requirement may not always be feasible in practice. To this end, transmission schemes with reduced feedback requirements ${ }^{3}$ have been developed [7], [14]-[16], where encoded packets instead of the native data packets are transmitted and only 1-bit acknowledgement (ACK) is required when a sufficient number of encoded packets is correctly received. In this section, we investigate how the above presented transmission algorithm can be applied in this modified system setup.

\section{A. Modified System Model}

We consider the modified system model depicted in Fig. 3. The difference compared with the system model illustrated in Fig. 1 is that the transmitter is additionally equipped with grouping and encoder blocks prior to the scheduler. Moreover, the receiver contains a decoder block. The functionality of these blocks is explained as follows.

Let $k$ be the maximum encoding length ${ }^{4}$ of the reduced feedback coding scheme employed by the encoding process. The grouping block selects from the queue a sequence of native data packets and divides it into $\rho+1$ batches of length $k_{i}$ with $k_{i} \leq k$ for $i=1, \ldots, \rho+1$. The selection of batch sizes, $k_{i}$, depends on the queue size when a batch is created; this issue is discussed in more detail in Section IV-B. Also, the reason for constructing $\rho+1$ batches instead of a single one, is explained in Section IV-B. Next, the encoding process produces encoded packets. Each encoded packet corresponds to a different data batch and is a random linear combination ${ }^{5}$ of the packets of the data batch to which it belongs [14]-[16]. Finally, the transmitter transmits at most $(\rho+1)$ encoded packets, one encoded packet from each data batch, through the OW and RF transmission subsystems.

\footnotetext{
${ }^{3}$ Reduced feedback transmission algorithms offer the benefit of reducing the rate of the feedback information or equivalently reducing the energy consuption at the receiver for transmitting feedback information. The latter may be important in applications with limited power supplies at the receiver.

${ }^{4}$ In practice, this parameter can be determined by the receiver's power supplies required for transmitting the feedback packets.

${ }^{5} \mathrm{We}$ consider that both the generator coefficients and the packets belong to a field of finite size (see [16] for more implementation details).
}

At the receiver end, the decoder collects the successfully received encoded packets that correspond to each data batch. When the successfully received packets from a particular batch is sufficient to permit recovery of the original native packets, i.e., the matrix consisting of the generator coefficients of the successfully received packets ${ }^{6}$ is invertible, it informs back the transmitter, through 1-bit ACK, and this data batch is removed from the queue. We will assume in the analysis that follows that the $i$ th data batch is removed from the queue when $k_{i}$ encoded packets that correspond to this data batch are successfully received. In principle, the expected number of encoded packets needed for successfully decoding a batch of $k$ packets may be slightly higher than $k$, depending on the inherent structure of the code and the encoding length. However, by increasing the encoding length [14], the field size of the generator coefficients used in the encoding process [15] or both, this difference can be considered as negligible.

Based on the above, the queue evolves as

$$
Q(t+1)=[Q(t)-\tilde{\mu}(t)]^{+}+R(t),
$$

where $R(t)$ is the number of new packets admitted into the queue in time-slot $t$ and $\tilde{\mu}(t)$ is defined as

$$
\tilde{\mu}(t)=\sum_{i \in \mathbb{D}} k_{i},
$$

where $\mathbb{D}$ denotes the set of the batches that have been successfully recovered.

For deriving the performance bounds, we additionally define a virtual queue, denoted as $Q^{f}(t)$. This queue represents the number of the encoded packets left to be transmitted in order to remove the data batches from $Q(t)$ plus the number of unprocessed data packets, i.e., packets of $Q(t)$ that do not belong to data batches, in slot $t$. Hence, it evolves as

$$
Q^{f}(t+1)=\left[Q^{f}(t)-\mu(t)\right]^{+}+R(t),
$$

where $\mu(t)$ is defined in (16). Furthermore, it follows from the definitions that

$$
0 \leq Q(t)-Q^{f}(t) \leq \sum_{i}^{\rho+1} k_{i} \leq(\rho+1) k,
$$

for all $t$.

\footnotetext{
${ }^{6}$ Such coefficients are obtained at the receiver either from the headers of the successfully received packets [7], [16] or by using identical random number generators at both the transmitter and the receiver [14].
} 


\section{B. Discussion on the System Model}

In this part we discuss certain aspects on the implementation of the system model described above.

1) The Length of Data Batches: As the queue length stochastically varies over the time, it is possible that at the time-slot when at least one data batch has been successfully recovered, the number of the unprocessed data packets of the queue is less than $k$. If $k_{i}$ is fixed and equal with $k$ for all $i=1, \ldots,(\rho+1)$, the transmitter has to wait until a sufficient number of data packets is admitted to the queue, resulting in additional transmission delays. These delays may become significant at low data rates, where new data packets arrive at the queue less frequently.

This issue is resolved as follows. When an ACK for a batch that is being processed is received, if the number of the unprocessed packets in the queue is more than or equal to $k$, the batch size for the next batch to be created will be $k$; otherwise, the transmitter will adapt the length for the next data batch to the current number of the unprocessed packets. This option requires that the receiver knows the number of packets that are contained in a batch; this number can be placed at the header of transmitted packets.

2) Transmitting From Multiple Data Batches: The reader may wonder why data packets are divided in $(\rho+1)$ batches instead of one batch. The reason we follow the first approach is the following. When transmitting encoded packets within a time-slot only from a single batch, there is a chance that $l$ encoded packets remain to be successfully received in order to remove the data batch from the queue, where $l<(\rho+1)$. If feedback was available at the transmitter at the beginning of the slot, the transmitter could send $\rho+1-l$ encoded packets from a new data batch. However, since the transmitter receives feedback only at the end of a slot, it will transmit $(\rho+1)$ encoded packets from the same data batch, resulting in some loss of throughput. This throughput loss can be made small by increasing $k$; however large $k$ implies large decoding delays and this may be undesirable in certain applications. The proposed transmission scheme with the multiple batches avoids this loss of throughput for any $k$.

\section{Reduced Feedback HTC Algorithm}

The HTC algorithm presented in Section III-C can be applied without changes in this modified system setup. The only extra requirement is that the $V$ parameter of the HTC algorithm needs to be larger than $(\rho+1) k$ in order to allow enough packets to be stored in the queue for creating the data batches. As it is stated in the next theorem, the long-term time-average throughput of the HTC algorithm can again be pushed to within $O\left(\frac{1}{V}\right)$ of the optimal value obtained through the STAT policy, ${ }^{7}$ with a tradeoff in the worst case queue backlog.

Theorem 4: Suppose a reduced feedback encoding transmission scheme with maximum length $k$ is applied at the transmitter and the HTC algorithm is implemented over all time-slots $t \in\{1,2,3, \ldots\}$. Assuming initial conditions $Q(0)=0$,

\footnotetext{
${ }^{7}$ Full-feedback is required in the implementation of the STAT policy for the accurate queue update.
}

$H_{1}(0)=H_{2}(0)=H_{3}(0)=0$ and a control parameter $V>0$, it holds that:

1) The worst case queue backlog is upper bounded by a constant $Q_{\max }$ for all $t$ given by

$$
Q(t) \leq Q_{\max }=(\rho+1) k+A_{\max }+V
$$

2) The long-term time average throughput utility achieved by the HTC algorithm is within $O\left(\frac{1}{V}\right)$ of the optimal value, i.e.,

$$
\bar{R} \geq v^{*}-\frac{1}{V}\left(\frac{B \mathbb{E}\left\{T^{2}\right\}}{\mathbb{E}\{T\}}+(\rho+1) k A\right),
$$

where $v^{*}$ denotes the optimal value of the objective in OPT and $A=\max \left[A_{\max }, \rho+1\right]$.

Proof: For the complete proof of the theorem, please refer to the appendix.

The reduced feedback version of the HTC algorithm also satisfies the $O\left(\frac{1}{V}, V\right)$ utility-delay tradeoff for a given maximum encoding length $k$. Moreover, for a given throughput performance, it requires higher values for $V$ compared with the full feedback case, which increase as the maximum encoding length $k$ raises. Thus, a tradeoff between delay and feedback becomes apparent, since the increase of $k$ and $V$ correspond to feedback decrease and average delay increase, respectively.

\section{RESUlTS \& Discussion}

In this section, we present numerical results that illustrate the performance of the presented algorithm under various weather conditions. For deriving these results, we assume that the sets of available transmission power levels are $\mathcal{P}_{1}=\left\{0, P_{1, \text { max }}\right\}$ and $\mathcal{P}_{2}=\left\{0, P_{2, \max }\right\}$, while the maximum average total power consumption at the transmitter is equal with $\hat{P}=90 \mathrm{~mW}$. Furthermore, the functions $f_{1}(\cdot)$ and $f_{2}(\cdot)$ are assumed to be given by

$$
f_{1}\left(P_{1}(t)\right)=\frac{P_{1}(\tau)}{\eta_{1}} \quad \text { and } \quad f_{2}\left(P_{2}(t)\right)=\frac{P_{2}(\tau)}{\eta_{2}}
$$

where $\eta_{1}$ and $\eta_{2}$ denote the wall-plug efficiency of the laser [24] and the power efficiency factor of the RF transmitter [25], respectively, and assumed to be equal with $\eta_{1}=40 \%$ and $\eta_{2}=$ $50 \%$. The values of the remaining parameters for the OW and $\mathrm{RF}$ transmission subsystems and channel states are summarized in Tables I and II. Finally, we assume that $A_{\max }=10$ packets.

Fig. 4 illustrates the average throughput achieved by the HTC transmission algorithm when a single state channel process is assumed. Specifically, the average number of the packets admitted in the queue, $\bar{R}$, is plotted with respect to the mean rate of the arrival process, assuming $V=100$ and various states that correspond to clear air, moderate rain or haze weather conditions, i.e., $\mathcal{S}=\left\{s_{i}\right\}$ with $i=1,2,3$. As benchmarks, the average throughput achieved by the RF-only and OW-only systems, i.e., the RF and OW subsystems respectively that operate distinctly and have the same average transmission and total power constraints with the hybrid transmitter, as well as the throughput of the STAT policy defined in Lemma 1, are 


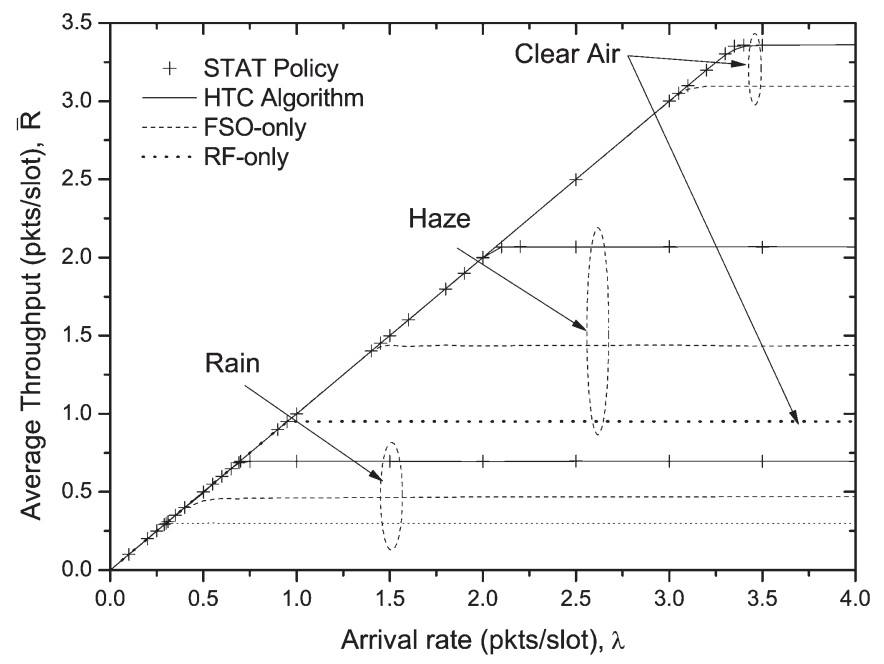

Fig. 4. Average throughput of a single channel state model.

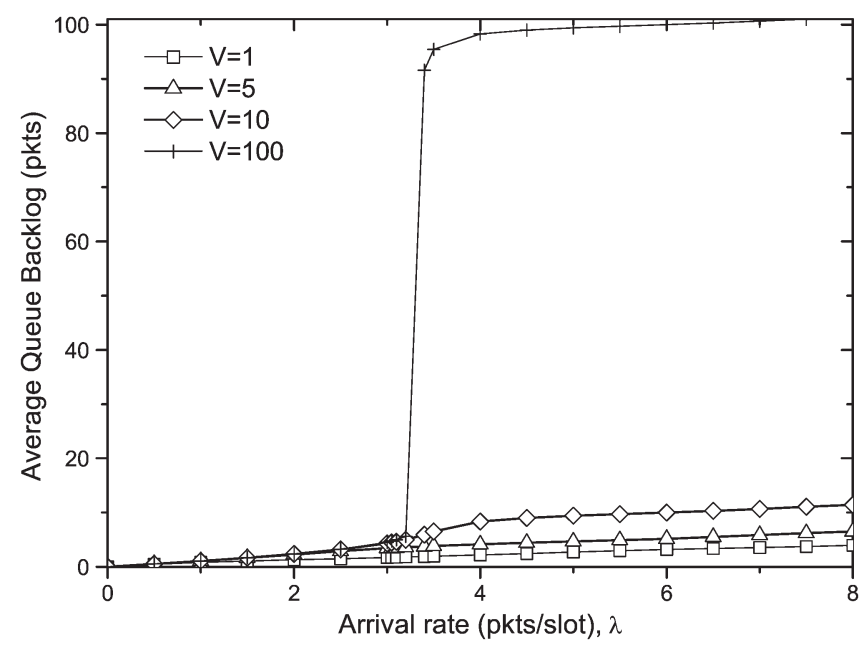

Fig. 5. $V$ parameter effect on the average queue size for clear air weather conditions.

plotted in the same graph. It can be observed that the throughput achieved by the HTC algorithm, which increases linearly with respect to $\lambda$ until a certain threshold $v^{*}$ which is the maximum rate that can be served by the system, is identical with the STAT policy throughput, in all cases examined. This happens due to the $V$ parameter value, which is high compared with $B$ and causes the error in (43) to be sufficiently reduced. Furthermore, when compared with the throughput achieved by the OWonly and RF-only systems, the HTC algorithm enhances the achievable throughput region of the hybrid transmitter without any additional power. This clearly illustrates that the proposed transmission scheme efficiently exploits both OW and RF links.

The effects of $V$ parameter on the performance of the HTC algorithm are investigated in terms of average throughput and queue size in Figs. 5 and 6, assuming single state channel process with clear air weather conditions, i.e., $\mathcal{S}=\left\{s_{1}\right\}$. As it can be observed in Fig. 5, the increase of $V$ results in the average queue backlog increase, which induces high packet transmission delays according to the Little's theorem. ${ }^{8}$ On the

\footnotetext{
${ }^{8}$ The average delays refer to packets that are admitted to the queue by the admission control.
}

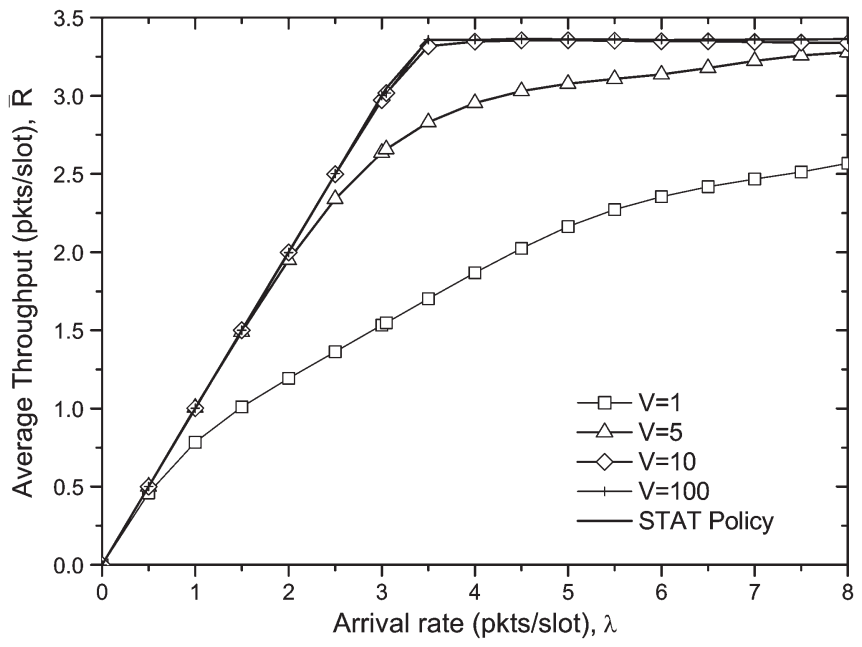

Fig. 6. $V$ parameter effect on the achieved throughput for clear air weather conditions.

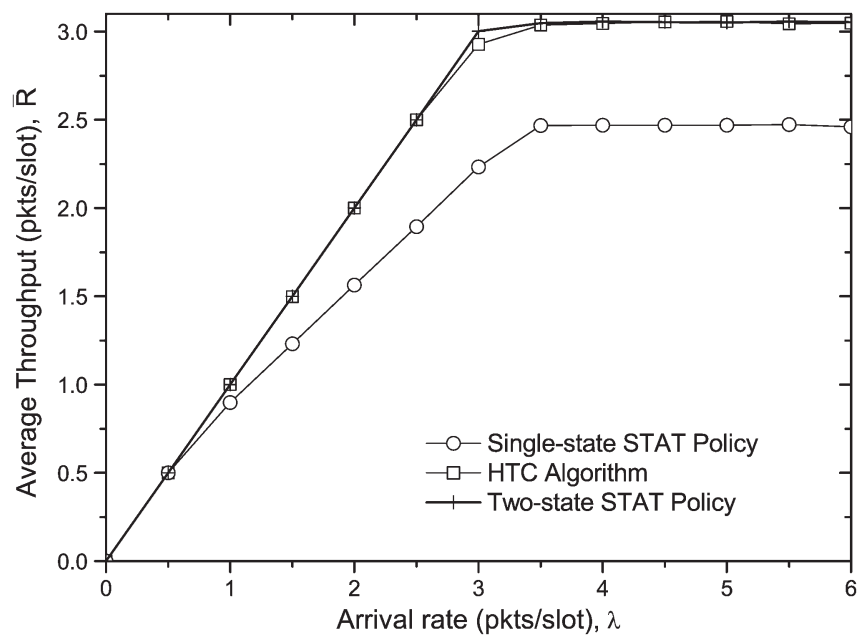

Fig. 7. Average throughput of a two state channel model.

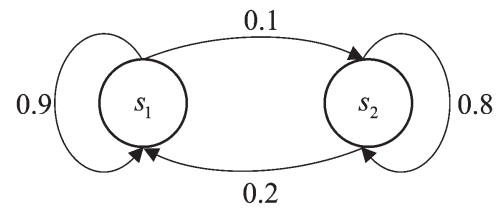

Fig. 8. Markov chain of a two state channel process.

other hand, Fig. 6 illustrates that the increase of $V$ improves the throughput achieved by the HTC algorithm, pushing it arbitrarily close to that of the optimal stationary policy. Thus, the theoretical $O\left(\frac{1}{V}, V\right)$ utility-delay tradeoff is verified.

Fig. 7 illustrates the performance of the HTC algorithm assuming a two state channel process with state space $\mathcal{S}=$ $\left\{s_{1}, s_{2}\right\}$, evolving according to the Markov chain ${ }^{9}$ of Fig. 8 . In the graph, the performance of the policy that observes the

\footnotetext{
${ }^{9}$ The Markov chain model is used for modeling weather conditions, no matter how fast these change (low values for the transition probabilities between the states of the Markov chain model slowly varying weather conditions). The Markov chain model can also be used for modeling more composite channel effects (such as weather changes in conjunction with movement).
} 


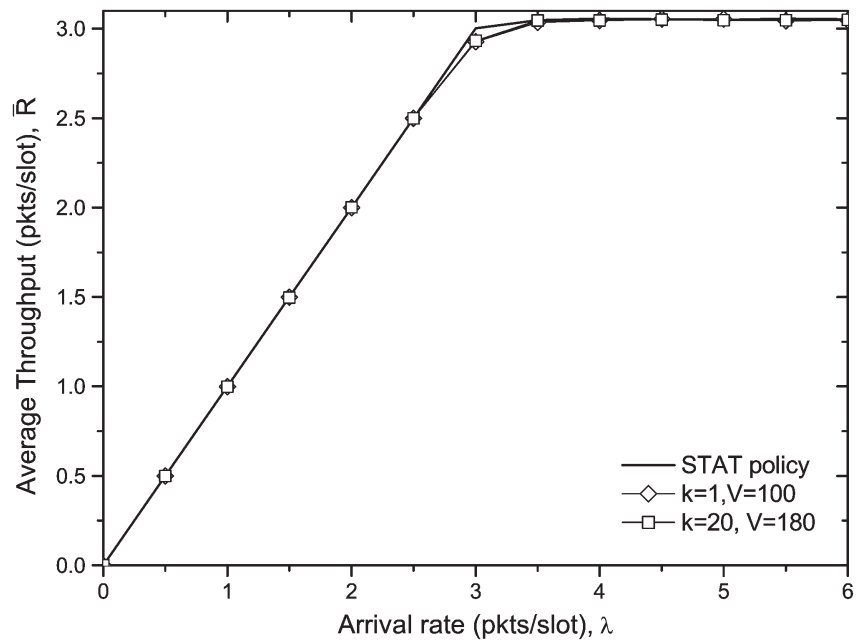

Fig. 9. Average throughput of reduced feedback HTC algorithm.

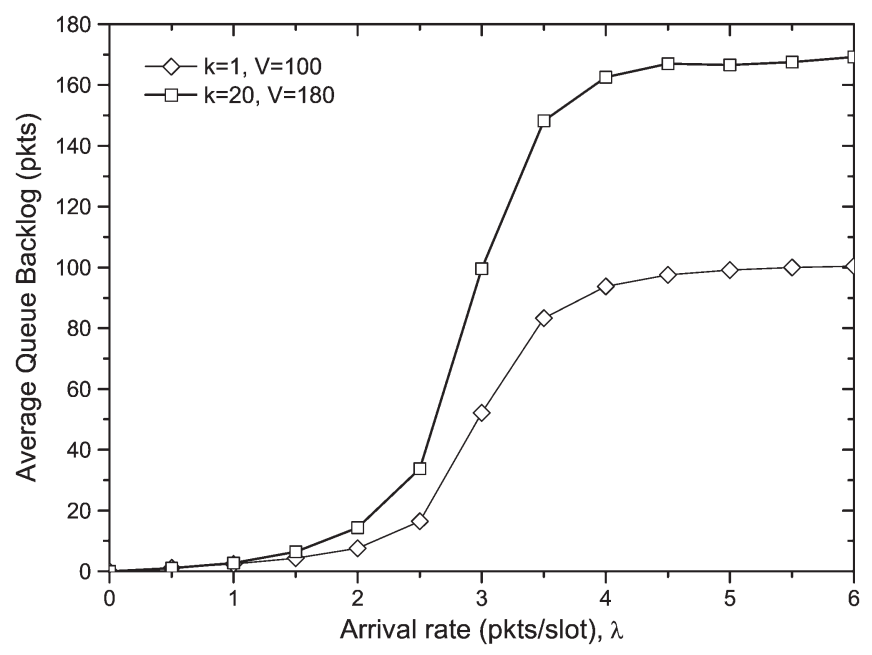

Fig. 10. Average queue backlog of reduced feedback HTC algorithm.

channel state and applies the single-state STAT policy in each state, i.e., the stationary policy derived by Lemma 1 assuming single state channel process, and the two-state STAT policy derived by Lemma 1 assuming that the transition probabilities of the Markov chain are known, are also plotted. It is obvious that the HTC algorithm achieves substantial throughput increase compared with the single-state STAT policy. This was expected since the latter policy does not take the structure of the Markov chain into account. Furthermore, when compared with the two-state STAT policy, the HTC algorithm with $V=100$ achieves almost identical throughput. It should be noted that in contrast with the two-state STAT policy, the HTC algorithm does not require any knowledge of the structure of the Markov chain, which in many cases it may be hard or impossible to be obtained. This poses as one of the main advantages of the proposed algorithm.

Finally, Figs. 9-11 illustrate the performance of the reducedfeedback HTC algorithm assuming the channel model Markov chain of Fig. 8. Specifically, the average throughput, the average queue backlog and the mean feedback rate, i.e., the average number of ACKs sent back to the transmitter, of the reducedfeedback HTC algorithm are investigated for two different

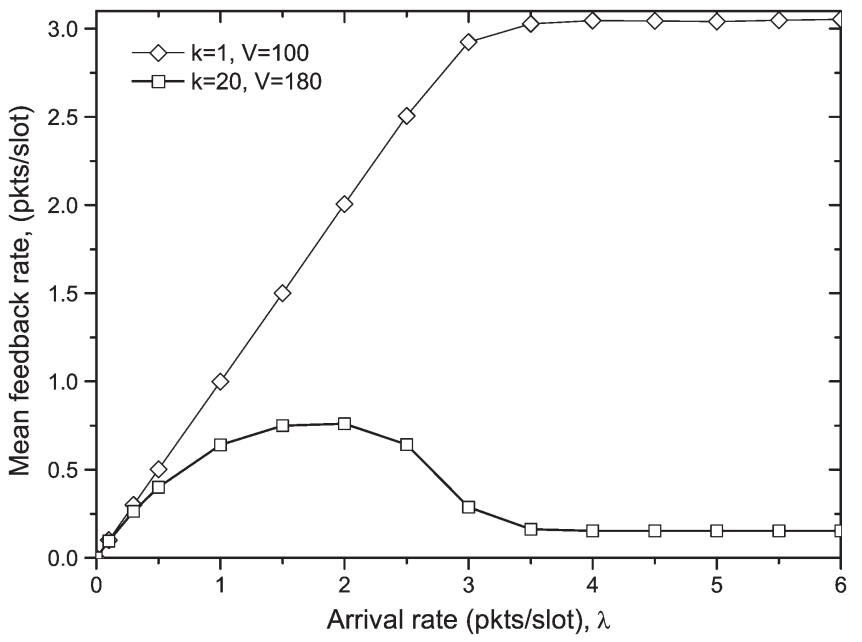

Fig. 11. Feedback requirements of reduced feedback HTC algorithm.

sets of parameters $k$ and $V, k=1 V=100$ (corresponds to full feedback) and $k=20, V=180$. It can be verified from Fig. 9 that the throughput achieved by the HTC algorithm is almost identical for both sets of parameters chosen and close to the performance of the STAT policy. When comparing the average queue backlog in Fig. 10, the average queue backlog that corresponds to $k=20$ and $V=180$ is higher than the average backlog that corresponds to the full-feedback case. This was expected due to the maximum queue size bound derived in (48). On the other hand, Fig. 11 demonstrates that the set of parameters that corresponds to higher average queue backlog has less feedback requirements, thus, revealing the tradeoff of the reduced-feedback HTC algorithm between feedback and delay. It should be noted that for low data rates (the regime with $\lambda<$ 0.5 ) the feedback requirements are the same, irrespective of the values chosen for the parameters $k$ and $V$. This is explained as follows. At low data rates there are not enough packets for creating full length data batches and the encoding length is influenced only by the input data rate. However, as the input data rate increases and approaches $v^{*}$, the possibility that there are enough packets to create full-length data batches increases and the effective encoding length approaches $k$. At this data rate regime, the merits of reducing feedback requirements as $k$ is increased, become more apparent.

\section{CONCLUSION}

We developed a transmission algorithm for hybrid OW/RF systems that maximizes their throughput subject to the transmitter per-link and total average power constraints. We used the Lyapunov Optimization technique to design an online flow control and resource allocation algorithm, which provides tight guarantees in terms of power consumption, while its throughput performance can be pushed arbitrarily close to the optimal value with a trade-off in the average delay. The major advantage of the proposed transmission algorithm is that it does not require knowledge of the underlying Markov chain of the channel process, or the statistics of the packet arrival process; it only needs to observe the channel statistical parameters in each time-slot, and based on the queue size, it takes the appropriate decisions. 


$$
\begin{aligned}
\Delta\left(\Theta^{f}\left(t_{r}\right)\right) & -V \mathbb{E}\left\{\sum_{\tau=t_{r}}^{t_{r}+T_{r}-1} R(\tau) \mid \Theta^{f}\left(t_{r}\right)\right\} \leq B \mathbb{E}\left\{T_{r}^{2} \mid \Theta^{f}\left(t_{r}\right)\right\}+Q\left(t_{r}\right) \mathbb{E}\left\{\sum_{\tau=t_{r}}^{t_{r}+T_{r}-1}(R(\tau)-\mu(\tau)) \mid \Theta^{f}\left(t_{r}\right)\right\} \\
& -V \mathbb{E}\left\{\sum_{\tau=t_{r}}^{t_{r}+T_{r}-1} R(\tau) \mid \Theta^{f}(t)\right\}+\left(Q^{f}\left(t_{r}\right)-Q\left(t_{r}\right)\right) \mathbb{E}\left\{\sum_{\tau=t_{r}}^{t_{r}+T_{r}-1}(R(\tau)-\mu(\tau)) \mid \Theta^{f}\left(t_{r}\right)\right\} \\
& +H\left(t_{r}\right) \mathbb{E}\left\{\sum_{\tau=t_{r}}^{t_{r}+T_{r}-1}\left(f_{1}\left(P_{1}(\tau)\right)+f_{2}\left(P_{2}(\tau)\right)-\hat{P}\right) \mid \Theta^{f}\left(t_{r}\right)\right\}
\end{aligned}
$$

$$
\begin{aligned}
\Delta\left(\Theta^{f}\left(t_{r}\right)\right) & -V \mathbb{E}\left\{\sum_{\tau=t_{r}}^{t_{r}+T_{r}-1} R(\tau) \mid \Theta^{f}\left(t_{r}\right)\right\} \leq B \mathbb{E}\left\{T_{r}^{2} \mid \Theta^{f}\left(t_{r}\right)\right\}-V \mathbb{E}\left\{\sum_{\tau=t_{r}}^{t_{r}+T_{r}-1} R(\tau) \mid \Theta^{f}(t)\right\} \\
& +Q\left(t_{r}\right) \mathbb{E}\left\{\sum_{\tau=t_{r}}^{t_{r}+T_{r}-1}(R(\tau)-\mu(\tau)) \mid \Theta^{f}\left(t_{r}\right)\right\}+(\rho+1) k A \mathbb{E}\left\{T_{r} \mid \Theta^{f}\left(t_{r}\right)\right\} \\
& +H\left(t_{r}\right) \mathbb{E}\left\{\sum_{\tau=t_{r}}^{t_{r}+T_{r}-1}\left(f_{1}\left(P_{1}(\tau)\right)+f_{2}\left(P_{2}(\tau)\right)-\hat{P}\right) \mid \Theta^{f}\left(t_{r}\right)\right\}
\end{aligned}
$$

Furthermore, in order to alleviate the requirement for full feedback at the transmitter, we modified the proposed algorithm to comply with hybrid OW/RF systems employing reducedfeedback coding schemes. It was shown that the throughput performance of the modified transmission policy can also be pushed arbitrarily close to the optimal value, while a tradeoff between feedback requirements and average delay was revealed. An interesting direction for future work can be the design of link-layer policies that offer the optimal throughput and have additional requirements (such as minimal delay or jitter) as well.

\section{APPENDIX}

The first part of the theorem follows directly by combining (47) with the bound of (42), which refers to the transmitter queue when there is complete feedback.

The second part of the theorem 4 can be proved using the variable-slot "Drift Plus Penalty" expression, when the virtual queue $Q^{f}(t)$ is considered. After defining the system's backlog in this case as $\Theta^{f}(t)=\left\{Q^{f}(t), H(t)\right\}$, it follows, based on the analysis of Section III-C and (35), that the variable-slot "Drift plus Penalty" expression for $\Theta^{f}(t)$ at renewal frame $t_{r}$ is bounded by (51), shown at the top of the page.

Due to (47), it holds that

$$
\left|\left(Q^{f}\left(t_{r}\right)-Q\left(t_{r}\right)\right) \sum_{\tau=t_{r}}^{t_{r}+T_{r}-1}(R(\tau)-\mu(\tau))\right| \leq(\rho+1) k T_{r} A
$$

with $A=\max \left[A_{\max }, \rho+1\right]$. Hence, (51) can be rewritten as (53), shown at the top of the page, which holds for any type of policies. By applying the reduced feedback HTC algorithm, the conditional expectations in the right-hand side of the above inequality are opportunistically minimized.
For deriving the performance bound of (49), we assume that a stationary policy is applied. Thus, the conditional expectations in the right-hand side of (53) are turned into pure expectations. Furthermore, assuming that the set of admissible policies is non-empty and employing the STAT policy defined in Lemma 1, (53) can be rewritten as

$$
\begin{aligned}
& \Delta\left(\Theta^{f}\left(t_{r}\right)\right)-V \mathbb{E}\left\{\sum_{\tau=t_{r}}^{t_{r}+T_{r}-1} R(\tau) \mid \Theta^{f}\left(t_{r}\right)\right\} \leq B \mathbb{E}\left\{T^{2}\right\} \\
& -V \mathbb{E}\{T\} v^{*}+(\rho+1) k A \mathbb{E}\{T\},
\end{aligned}
$$

where it has been used, derived based on the renewal-reward theorem [23, pp. 171], that

$$
\mathbb{E}\left\{\sum_{\tau=t_{r}}^{t_{r}+T_{r}-1} R^{\text {stat }}(\tau)\right\}=\mathbb{E}\{T\} \bar{R}^{\text {stat }}=\mathbb{E}\{T\} v^{*} .
$$

Taking the expectations of the above, summing the resulting telescoping series over $r \in\left\{0, \ldots, r^{*}-1\right\}$ and dividing by $V r^{*} \mathbb{E}\{T\}$, yields

$$
\begin{array}{r}
\frac{\mathbb{E}\left\{L\left(\Theta\left(t_{r^{*}}\right)\right)\right\}-\mathbb{E}\{L(\Theta(0))\}}{V \mathbb{E}\{T\} r^{*}}-\frac{\mathbb{E}\left\{\sum_{\tau=0}^{t_{r^{*}-1}} R(\tau)\right\}}{\mathbb{E}\{T\} r^{*}} \\
\leq \frac{B \mathbb{E}\left\{T^{2}\right\}}{\mathbb{E}\{T\} V}-v^{*}+\frac{k A(\rho+1)}{V}
\end{array}
$$

Due to the strong law for renewal processes [23, pp. 163], (49) follows from (56).

\section{REFERENCES}

[1] L. Andrews, R. L. Philips, and C. Y. Hopen, Laser Beam Scintillation With Applications. Bellingham, WA, USA: SPIE Press, 2001.

[2] H. Willebrand and B. S. Ghuman, Free Space Optics: Enabling Optical Connectivity in Today Networks. Indianapolis, IN, USA: Sams, 2002.

[3] S. Bloom and D. J. T. Heatley, "The last mile solution: Hybrid FSO radio," AirFiber Inc., Superior, WI, USA, White Paper 802-0008-000 M-A1, pp. 1-20, May 2002. 
[4] H. Wu and M. Kavehrad, "Availability evaluation of ground-to-air hybrid FSO/RF links," Int. J. Wireless Inf. Netw., vol. 14, no. 1, pp. 33-45, Mar. 2007.

[5] F. Nadeem et al., "Weather effects on hybrid FSO/RF communication link," IEEE J. Sel. Areas Commun., vol. 27, no. 9, pp. 1687-1697, Dec. 2009.

[6] D. K. Borah, A. C. Boukouvalas, C. C. Davis, and S. H. H. Yiannopoulos, "A review of communication-oriented optical wireless systems," EURASIP J. Wireless Commun. Netw., vol. 2012, no. 1, pp. 91:1-91:28, Mar. 2012.

[7] W. Zhang, S. Hranilovic, and C. Shi, "Soft-switching hybrid FSO/RF links using short-length Raptor-codes: Design and implementation," IEEE J. Sel. Areas Commun., vol. 27, no. 9, pp. 1698-1708, Dec. 2009.

[8] S. Deng, J. Liao, Z. R. Huang, M. Hella, and K. Connor, "Wireless connections of sensor network using RF and free space optical links," in Proc. SPIE, vol. 6303, 2007, p. 677307.

[9] F. Nadeem, S. Chessa, E. Leitgeb, and S. Zaman, "The effects of weather on the life time of wireless sensor networks using FSO/RF communication," Radioengineering, vol. 19, no. 2, pp. 262-270, Jun. 2010.

[10] D. Kedar, D. Grace, and S. Arnon, "Laser nonlinearity effects on optical broadband backhaul communication links," IEEE Trans. Aerosp. Electron. Syst., vol. 46, no. 4, pp. 1797-1803, Oct. 2010.

[11] S. Das et al., "Requirements and challenges for tactical free-space lasercomm," in Proc. IEEE MILCOM, Nov. 2008, pp. 1-10.

[12] M. J. Neely, Stochastic Network Optimization With Application to Communication \& Queueing Systems. San Rafael, CA, USA: Morgan \& Claypool, Aug. 2010.

[13] L. Georgiadis, M. J. Neely, and L. Tassiulas, "Resource allocation and cross-layer control in wireless networks," Found. Trends Netw., vol. 1, no. 1, pp. 1-144, 2006.

[14] D. J. C. MacKay, "Fountain codes," Proc. Inst. Elect. Eng.-Commun., vol. 152, no. 6, pp. 1062-1068, Dec. 2005.

[15] M. M. A. Eryilmaz, A. Ozdaglar, and E. Ahmed, "On the delay and throughput gains of coding in unreliable networks," IEEE Trans. Inf. Theory, vol. 54, no. 12, pp. 5511-5524, Dec. 2000.

[16] P. A. Chou, Y. Wu, and K. Jain, "Practical network coding," in Proc. Annu. Allerton Conf. Commun. Control Comput., pp. 40-49.

[17] S. Hranilovic, Wireless Optical Intensity Channels. Berlin, Germany: Springer-Verlag, 2005.

[18] T. A. Tsiftsis, "Performance of heterodyne wireless optical communication systems over gamma-gamma atmospheric turbulence channels," Electron. Lett., vol. 44, no. 5, pp. 372-373, Feb. 2008.

[19] I. S. Gradshteyn and I. M. Ryzhik, Table of Integrals, Series, and Products, 6th ed. New York, NY, USA: Academic, 2000.

[20] B. He and R. Schober, "Bit-interleaved coded modulation for hybrid RF/FSO systems," IEEE Trans. Commun., vol. 57, no. 12, pp. 3753-3763, Dec. 2009.

[21] M. K. Simon and M.-S. Alouini, Digital communications Over Fading Channels. Hoboken, NJ, USA: Wiley Interscience, 2005.

[22] D. Bertsimas and J. N. Tsitsiklis, Introduction to Linear Optimization. Belmont, MA, USA: Athena Scientific, 1997.

[23] R. Gallager, Discrete Stochastic Processes. Boston, MA, USA: Kluwer, 1996.

[24] G. P. Agrawal, Fiber-Optic Communication Systems. Hoboken, NJ, USA: Wiley, 2002.

[25] J. N. Murdock and T. S. Rappaport, "Consumption factor and powerefficiency factor: A theory for evaluating the energy efficiency of cascaded communication systems," IEEE J. Sel. Areas Commun., vol. 32, no. 12, pp. 1-16, Dec. 2014

[26] T. Kamalakis, I. Neokosmidis, A. Tsipouras, S. Pantazis, and I. Andrikopoulos, "Hybrid free space optical/millimeter wave outdoor links for broadband wireless access networks," in Proc. IEEE PIMRC, Sep. 2007, pp. 1-5.

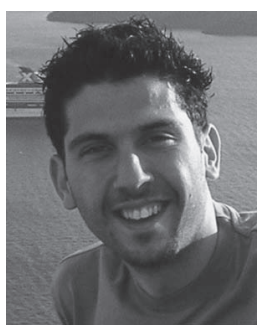

Nestor D. Chatzidiamantis (S'08-M'14) was born in Los Angeles, CA, USA, in 1981. He received the Diploma degree (5 years) in electrical and computer engineering from Aristotle University of Thessaloniki, Greece, and the M.S. award (with Distinction) in telecommunication networks and software from the University of Surrey, U.K., in 2005 and 2006, respectively. In April 2012, he received the $\mathrm{Ph} . \mathrm{D}$. degree from the ECE Department, Aristotle University, where he currently works as a Postdoctoral Research Associate.

His research areas span the performance analysis of wireless communication systems over fading channels, communications theory, cognitive radio, and free-space optical communications.

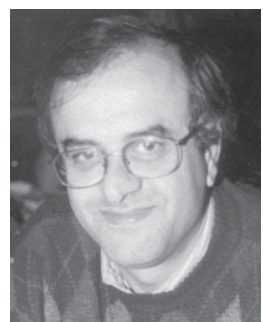

Leonidas Georgiadis (S'76-M'78-SM'96) received the Diploma degree in electrical engineering from Aristotle University, Thessaloniki, Greece, in 1979, and the M.S. and Ph.D. degrees, both in electrical engineering, from the University of Connecticut, in 1981 and 1986, respectively. From 1986 to 1987, he was Research Assistant Professor at the University of Virginia, Charlottesville, VA, USA. In 1987, he joined IBM T. J. Watson Research Center, Yorktown Heights as a Research Staff Member. Since October 1995, he has been with the Telecommunications Department, Aristotle University.

His interests are in the area of wireless networks, energy efficient communications, information theory and coding, distributed systems, routing, scheduling, congestion control, modeling and performance analysis.

In 1992, he received the IBM Outstanding Innovation Award for his work on goal-oriented workload management for multi-class systems and in 1994 the IBM Research Division Award for work on the architecture and design of NBBS (Broadband Networking Systems).

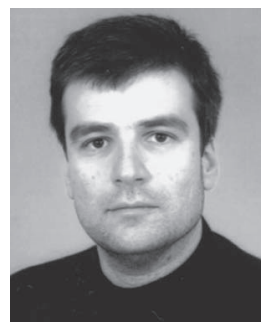

Harilaos G. Sandalidis received the five-year Diploma degree in electronics and computer engineering and the M.Sc. degree in business administration from the Production Engineering and Management Department, Technical University of Crete, Chania, Greece, in 1995 and 1998, respectively, and the M.Sc. degree in radio-frequency and microwave communications and the Ph.D. degree in the telecommunications area from the Electronics and Telecommunications (formerly Electronics and Electrical Engineering) Department, University of Bradford, Bradford, U.K., in 1996 and 2002, respectively.

Between 1996 and 2001, he was a Research Assistant at the Telecommunications Systems Institute of Crete, Chania, Greece. After his military service, he joined TEMAGON, the technology consulting branch of the Hellenic Telecommunications Organization (OTE Group), in 2002, where he was involved in the risk mitigation program for the 2004 Olympic Telecommunication Network in collaboration with Telcordia Technologies, Inc. In March 2009, he joined the University of Central Greece as a Lecturer. He is now an Assistant Professor at the Department of Computer Science and Biomedical Informatics, University of Thessaly, Lamia, Greece. His current research interests include optical wireless communications, visible light communications and wireless body area networking.

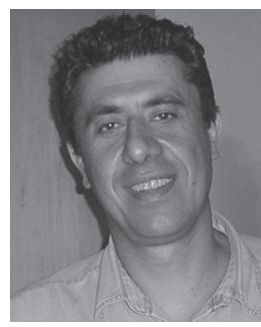

George K. Karagiannidis (M'96-SM'03-F'14) received the University Diploma and Ph.D. degree in electrical and computer engineering from the University of Patras in 1987 and 1999, respectively. From 2000 to 2004, he was a Senior Researcher at the Institute for Space Applications and Remote Sensing, National Observatory of Athens, Greece. In June 2004, he joined the faculty of Aristotle University of Thessaloniki, Greece, where he is currently a Professor in the Electrical \& Computer Engineering Department and Director of Digital Telecommunications Systems and Networks Laboratory.

His research interests are in the broad area of digital communications systems with emphasis on communications theory, energy efficient MIMO and cooperative communications, satellite communications, cognitive radio, localization, smart grid and optical wireless communications. He is the author or coauthor of more than 250 technical papers published in scientific journals and presented at international conferences. He is also author of the Greek edition of the book Telecommunications Systems and co-author of the book Advanced Optical Wireless Communications Systems (Cambridge Publications, 2012). $\mathrm{He}$ is co-recipient of the Best Paper Award of the Wireless Communications Symposium (WCS) in the IEEE International Conference on Communications (ICC07), Glasgow, U.K., June 2007.

Dr. Karagiannidis has been a member of Technical Program Committees for several IEEE conferences such as ICC, GLOBECOM, VTC, etc. In the past, he was Editor for Fading Channels and Diversity of the IEEE TRANSACTIONS ON COMMUNICATIONS, Senior Editor of IEEE COMMUNICATIONS LETTERS and Editor of the EURASIP Journal of Wireless Communications \& Networks. He was Lead Guest Editor of the special issue on Optical Wireless Communications of the IEEE Journal on SELECTED AREAS IN COMMUNiCATIONS and Guest Editor of the special issue on "Large-scale multiple antenna wireless systems." Since January 2012, he is the Editor-in Chief of IEEE COMMUNICATIONS LETTERS. 